\title{
Entre Shakespeare et Montaigne : quelques nouveaux tours d'escrime
}

\section{Richard Hillman}

\section{(2) OpenEdition Journals}

Édition électronique

URL : http://journals.openedition.org/shakespeare/166

DOI : 10.4000/shakespeare.166

ISSN : 2271-6424

Éditeur

Société Française Shakespeare

Édition imprimée

Date de publication : 1 novembre 2004

Pagination : 135-153

ISBN : 2-9521475-0-7

Référence électronique

Richard Hillman, «Entre Shakespeare et Montaigne : quelques nouveaux tours d'escrime », Actes des congrès de la Société française Shakespeare [En ligne], 21 | 2004, mis en ligne le 31 janvier 2007, consulté le 02 mai 2019. URL : http://journals.openedition.org/shakespeare/166 ; DOI : 10.4000/ shakespeare.166 


\section{Shakespeare et Montaigne vers un nouvel humanisme}

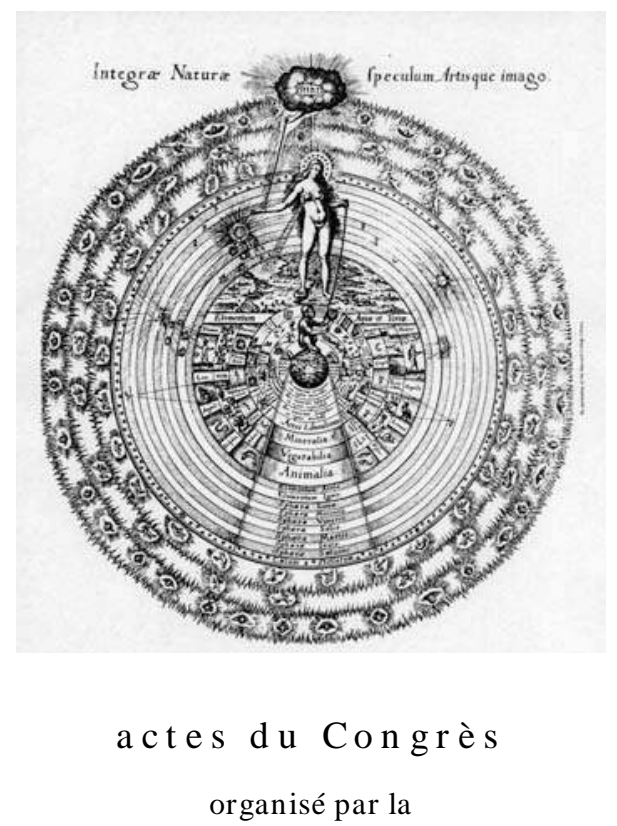

SOCIÉTÉ FR ANÇAISE SH AKESPEARE en collaboration avec la

S OC I É T É I N T E R N A T I O N A L E DES AM I D E M O N A I G N les 13,14 et 15 mars 2003

$$
\begin{gathered}
\text { textes réunis par } \\
\text { Pierre KAPITANIAK } \\
\text { sous la direction de } \\
\text { Jean-Marie MAGUIN }
\end{gathered}
$$




\section{COMITÉ SCIENTIFIQUE :}

Margaret Jones-Davis

Gisèle Venet

Jean-Marie Maguin

Yves Peyré

François Laroque

Pierre Kapitaniak

\section{COUVERTURE :}

Robert Fludd

Utriusque Cosmi Historia (1617-19)

planche 17

conception graphique et logo

Pierre Kapitaniak

\section{(C) 2003 Société Française Shakespeare}

Institut du Monde Anglophone

Université de Paris III - Sorbonne Nouvelle

http:// univ-montp3.fr/SFS/

5 rue de l'École de Médecine

75006 Paris

Diffusion :

AVL DIFFUSION

Parc Euromédecine

34198 MONTPELLIER CEDEX 5

ISBN 2-9521475-0-7

Tous droits de traduction, de reproduction et d'adaptation réservés pour tous les pays 


\title{
ENTRE SHAKESPEARE ET MONTAIGNE : QUELQUES NOUVEAUX TOURS D'ESCRIME
}

\author{
Richard HILLMAN
}

\begin{abstract}
En général, les nombreux critiques qui se sont penchés sur l'influence de Montaigne sur Shakespeare ont basé des correspondances intellectuelles plus ou moins vastes sur des échos plus ou moins menus. lls ont été peu concernés par la dynamique d'interprétation qui aurait pu être déclenchée chez les spectateurs contemporains qui entendaient ces échos. Cette communication cherche à récupérer une telle dynamique en appliquant à l'expérience théâtrale la notion, fondée sur la théorie de l'intertextualité, qu'une anomalie dans la " grammaire " d'un texte peut signaler la présence plus ou moins " déroutante " d'un intertexte. Ainsi dans quelques cas particuliers le texte théâtral de Shakespeare se révèle comme engagé dans un jeu avec celui de Montaigne, jeu qui produit des significations insoupçonnées tout en mettant en relief à la fois la dette et l'originalité du dramaturge anglais.

Most of the numerous critics who have considered the influence of Montaigne on Shakespeare have approached the subject by establishing general intellectual affinities on the basis of specific verbal parallels. They have been little concerned with the interpretative dynamic that might have been set in motion for those members of contemporary audiences who also heard such echoes. This paper attempts to reconstruct such a dynamic by applying to the dramatic experience the notion, current in intertextual theory, that a deviation from the "grammar" of a text may point to the more or less disruptive presence of an intertext. In this light, Shakespeare's text sometimes reveals itself as engaged in a virtual fencing-match with that of Montaigne, a process that generates unexpected meanings and highlights both the indebtedness and the originality of the English playwright.
\end{abstract}

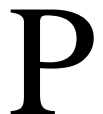

ar ce titre j'entends une relation qui est en même temps un espace intertextuel, l'espace d'un jeu entre deux textes qu'il convient d'appeler, pourquoi pas, par les noms de leurs auteurs. Et pourquoi ne pas imaginer ce jeu comme un match d'escrime, organisé autour des «agrammaticalités » qui activent, chez le spectateur ou le lecteur, une dynamique quasi-oppositionnelle, composée de moments de contact et de désengagement, d'action et de réaction? J'ai recours à l'intertextualité comme alternative à la méthode traditionnelle de la critique pour tracer l'influence de Montaigne sur Shakespeare, méthode qui consiste à compiler des correspondances verbales ou intellectuelles plus ou moins précises. Je me base plutôt sur l'idée que, pour un contemporain de Shakespeare qui connaissait Montaigne, la présence de ce dernier chez Shakespeare pouvait se faire sentir sous forme de déformation et en termes d'engagement plutôt que d'emprunt. 
Pour qu'une « agrammaticalité » opère (le terme est de Michael Riffaterre $\left.{ }^{218}\right)$, il faut qu'il y ait une « grammaire», ce qui met en valeur la subjectivité des démarches. Une « grammaire» textuelle n'existe que dans l'esprit d'un «lecteur» et peut exister sur plusieurs registres : pour le présent exercice j'ai choisi le registre du genre, sur la prémisse que Shakespeare communiquait avec son public surtout en utilisant, et souvent en coupant à travers, des codes génériques.

Dans un premier temps, j'aborde le cas quasi-classique de Hamlet, où un nombre important de correspondances verbales quelques-unes définitives et plusieurs suggestives ${ }^{219}$ - établit que Shakespeare a puisé dans les Essais, du moins dans la traduction de Florio (sous forme manuscrite avant 1603), mais peut-être aussi dans l'original. La plupart de ces correspondances appartiennent aux réflexions dites «philosophiques»du personnage principal, jusqu'au point où la pensée de Hamlet semble marquer l'influence par excellence des Essais et surtout celle de l'«Apologie de Raymond Sebond ", chapitre qui oppose de façon soutenue les deux perspectives sur l'être humain - comme «beauty of the world» et «quintessence of dust» (II.ii.307-8) - mises en opposition par Hamlet. C'est justement l'ironie de cette juxtaposition qui, pour Robert Ellrodt dans son article

218 Voir notamment les articles suivants de Michael Riffaterre: "Sémiotique intertextuelle: l'interprétant», Revue d'esthétiques, vol. 1-2, 1979, p. 134 ; «Syllepsis», Critical Inquiry, vol. 6, 1980, p. 627 ; «L'intertexte inconnu », Littérature, vol. 41, 1981, p. 5.

${ }^{2} 19$ La liste de correspondances proposée est longue, et comme le manque de consensus le constate, ces catégories seront toujours subjectives (celle de «décisif » remonte au moins jusqu'à John M. Robertson, Montaigne and Shakespeare, and Other Essays on Cognate Questions, 2e éd., Londres, Black, 1909, p. 34). D'où l'avantage de la théorie intertextuelle, qui (sauf pour certains praticiens tels que Michael Riffaterre) est capable d'intégrer la subjectivité. Parmi les échos qui continuent à résonner pour la plupart des spécialistes on pourrait peut-être citer le parallèle entre «divinty [...] rough-hew » (v.ii.10-11) et « roughly hew [...] heaven » (Montaigne, III, 8; trad. Florio, 3: 171), ainsi que celui entre «consummation [...] dreams» (III.i.63-66) dans le soliloque «To be...» de Hamlet et Montaigne III, 12, trad. Florio, 3:309, sur la mort : «If it be a consummation of one's being, it is also an amendment and entrance into a long and quiet night. We find nothing so sweet in life, as a quiet rest and gentle sleep, and without dreams ». Voir Hamlet, éd. Harold Jenkins, coll. "Arden Shakespeare», Londres, Methuen, 1982, p. 557 et 489 - mon édition de référence ici. Le premier parallèle est particulièrement important, par exemple, pour Robert Ellrodt, "Self-Consciousness in Montaigne and Shakespeare », Shakespeare Survey, vol. 28, 1975, p. 403, le second pour Harry Levin dans The Question of Hamlet, New York, Oxford University Press, 1959, p. 72-73. Dans le présent article je cite les Essais, en suivant les usages normaux, dans l'édition de Pierre Villey, révisée par V.-L. Saulnier, 3 e éd., Paris, Presses Universitaires de France, 1978, et leur traduction par John Florio, en donnant le numéro de volume et page, dans Montaigne's Essays, 3 vol., Everyman's Library, Londres, Dent et New York, Dutton, 1910 
indispensable, «discloses a kinship in spirit not traceable in the other analogues $»^{220}$.

Montaigne établit vers le début de l'«Apologie » la fragilité de l'idée que «ce branle admirable de la voute céleste [this admirable moving of heavens vault] » (II, 12, 450 a ; trad. Florio, $2: 139)$ à été fait pour l'homme, «cette misérable et chetive creature [this miserable and wreched creature] » qui se croit «estre seul, en ce grand bastimant, qui ayt la suffisance d'en recognoistre la beauté et les pièces [to be the onely absolute creature in this huge worlds-frame, perfectly able to know the absolute beautie, and severall parts thereof] ». Voilà l'illusion qui semble s'être écroulée chez Hamlet, qui explique à Rosencrantz et Guildenstern qu'il a dernièrement «lost all my mirth, foregone all custom of exercises » (II.ii.296-97) et qu'à travers sa mélancolie, «this goodly frame the earth seems to me a sterile promontory, [...] this brave o'erhanging firmament [...] a foul and pestilent congregation of vapours » (II.ii.298-303), tandis qu'il sait bien à quel point l'homme est un «piece of work» sans pareil :

how noble in reason, how infinite in faculties, in form and moving how express and admirable, in action how like an angel, in apprehension how like a god: the beauty of the world, the paragon of animals. (II.ii.303-7)

Il y a dans ces propos un glissement notable du vocabulaire de Montaigne et/ou Florio de la création («huge world's-frame», «admirable moving», «beauty» - même «pièces») vers la créature même, ce qui illustre à merveille l'argument de l'essayiste sur la vanité humaine. Mais les manœuvres autour de ces propos illustrent aussi le paradoxe de l'argumentation montaignienne. Hamlet représente sa pensée pessimiste comme une déviation de la raison, alors qu'il est en train de se servir justement de sa raison, dont d'ailleurs il est actuellement question, comme une arme contre ses adversaires. Jouer avec la raison contre la raison, c'est la démarche essentielle de l'« Apologie », mais au fond n'est-ce pas jouer contre soi-même?

Pour Hamlet ici, comme dans des soliloques où il joue plus ouvertement contre lui-même, c'est la raison, assimilée au discours, qui élève l'homme au-dessus des bêtes et l'approche du divin : "a beast that wants discourse of reason »(I.ii.150); «A beast, no more. / Sure

220 Ellrodt, p. 40. 
he that made us with such large discourse, / [...] gave us not / That capability and godlike reason / To fust in us unus'd» (IV.iv.35-39). La critique shakespearienne a dûment repéré ce lieu commun chez Montaigne, sans pour autant le placer au cœur du scepticisme de l'« Apologie», dont la prémisse est que «nos raisons et nos discours humains, c'est comme la matière lourde et sterile : la grace de Dieu en est la forme [our reason and humane discourse, is as the lumpish and barren matter; and the grace of God is the forme thereof] » (II, 12, 447a ; trad. Florio, 2: 136) : «sterile» comme l'est devenue la terre même pour Hamlet ${ }^{221}$.

En fait, c'est par ce biais, et en poursuivant une image hautement significative, que l'essayiste lance son défi à l'homme :

Considerons donq pour cette heure l'homme seul, sans secours estranger, armé seulement de ses armes, et despourveu de la grace et cognoissance divine, qui est tout son honneur, sa force et le fondement de son estre. [...] Qu'il me face entendre, par l'effort de son discours, sur quels fondamens il a basty ces grands avantages qu'il pense avoir sur les autres creatures.

[Let us now but consider man alone without other help, armed but with his own weapons, and unprovided of the grace and knowledge of God, which is all his honour, all his strength, and all the ground of his being. [...] Let him with the utmost power of his discourse make me understand, upon what foundation, he hath built those great advantages and ods, he supposeth to have over other creatures.] (II, 12, 449-50a; trad. Florio, $2: 139$ )

Effectivement, plus tard Hamlet se représentera ainsi dépourvu dans sa lettre à Claudius : "High and mighty, you shall know that I am set naked on your kingdom. [...] And in a postscript here he says "Alone" » (IV.vii.42-51). Il se déclarera même converti au principe montaignien (et paulinien) que «nostre sagesse n'est que folie devant Dieu [all our wisdome is but folly before God]» (II, 12, 449a; trad. Florio, 2 : $138)^{222}$, lorsqu'il dira à Horatio, à travers Montaigne/Florio, «There's a divinity that shapes our ends, / Rough-hew them how we will»

${ }^{221}$ Shakespeare n'emploie ce mot, qui ne figure pas dans la traduction de Florio, que rarement. Sur la tendance dans l'« Apologie» du lien entre «raison» et «discours » à rendre problématique la distinction entre l'homme et les animaux, voir Marie-Luce Demonet, "À plaisir». Sémiotique et scepticisme chez Montaigne, Orléans, Paradigme, 2003 , p. 81 et seq.

222 Voir 1 Cor. 3 : 19 
$(\text { v.ii.10-11 })^{223}$. Mais à ce moment il va également se vanter du stratagème hyper-rationnel par lequel il s'est débarrassé de Rosencrantz et Guildenstern, alors que son acceptation de l'invitation au match d'escrime ne nous convaincra pas qu'il sait bien distinguer entre la sagesse et la folie. Après tout, si « l'homme qui présume de son sçavoir, ne sçait pas encore que c'est que sçavoir [man, who presumeth of his knowledge, doth not yet know what knowledge is] » (II, 12, 449a ; trad. Florio, $2: 138)^{224}$, comment différencier l'espoir et le désespoir? Voilà un argument également adapté au suicide qu'à la foi. Dans ce contexte, s'en remettre à «a divinity that shapes our ends» ou à «a special providence in the fall of a sparrow» (v.ii.215-16) revient à se donner au néant : «Since no man of aught he leaves, knows, what is't to leave betimes? » (v.ii.218-20 $)^{225}$.

Nous sommes tellement habitués à Hamlet penseur que nous risquons de ne pas nous rendre compte de la désorientation probable des premiers auditeurs. Ce n'est pas le commentaire continu sur ses griefs et sa vengeance qui les aurait surpris : ces tendances rhétoriques

\footnotetext{
223 Voir supra, note 2.

224 Voir 1 Cor. $8: 2$.

225 Ici j'adapte la version du $2^{\mathrm{e}}$ in-quarto, dont Jenkins (p. 565-66) régularise la grammaire sans raison suffisante, à mon avis, tout en faisant un argument généralement convaincant contre la version du $1^{\text {er }}$ in-Folio («ha's ought of what he leaues »). Sur un tel point textuel il ne peut y avoir de certitude, mais la conjonction dans l'«Apologie» des concepts de l'ignorance humaine fondamentale, du suicide, et d'un duel me paraît assez concluante. Vers la fin de l'essai, au moment crucial où ces deux derniers éléments se réalisent pleinement, Montaigne revient à la première :

Vrayement Protagoras nous en comtoit de belles, faisant l'homme la mesure de toutes choses, qui ne sceut jamais seulement la sienne. [...] Or, luy estant en soy si contraire et l'un jugement en subvertissant l'autre sans cesse, cette favorable proposition n'estoit qu'une risée qui nous menoit à conclurre par necessité la néantise du compas et du compasseur.

Quand Thales estime la cognoissance de l'homme tres difficile à l'homme, il luy apprend la cognoissance de toute autre chose luy estre impossible.
}

[Truely Protagoras told us prettie tales, when hee makes man the measure of all things, who never knew so much as his owne. [...] Now he being so contrary in himselfe, and one judgement so uncessantly subverting another, this favorable proposition was but a jest, which indused us necessarily to conclude the nullity of the Compasse and the Compasser. When Thales judgeth the knowledge of man very hard unto man, hee teacheth him the knowledge of all other things to be impossible unto him.] (II, 12, 557c; trad. Florio, $2: 270)$

Ironiquement, d'autres critiques ont fondé sur un passage de Montaigne (I, 19) très proche de Measure for Measure, III.i.32 et seq., un argument en faveur de « has ». Voir J acob Feis, Montaigne and Shakespeare, and Other Essays on Cognate Questions (1884 ; ré-éd. New York, AMS, 1970), p. 111, et Elizabeth Robbins Hooker, «The Relation of Montaigne to Shakespeare », PMLA, vol. 17, 1902, p. 319-20. 
sont déjà présentes dans le récit de Belleforest, tandis que la mention satirique par Nashe de «whole Hamlets [...] of tragicall speeches » 226 mène à croire qu'elles abondaient aussi dans le soi-disant Ur-Hamlet, vraisemblablement de Thomas Kyd. Pour en juger par The Spanish Tragedy, du même dramaturge, la pièce précédente aurait bien pu exploiter aussi l'imputation de la folie au héros. Mais rien n'indique que dans une version antérieure quelconque, le héros ne poursuit pas, à travers des obstacles bien concrets, le parcours vindicatif essentiel à la « grammaire» du genre ${ }^{227}$.

Or, chez Shakespeare le «spectacle» qui devrait à la fois dévoiler et punir le criminel est déplacé vers le milieu de la pièce et ne sert plus ni l'une ni l'autre de ces fonctions. Le héros accepte d'être embarqué pour l'étranger au moment où il se déclare enfin prêt à agir. Surtout le complot par lequel sa vengeance s'accomplit n'est pas le sien mais celui de son ennemi, et sa participation entraîne un pressentiment de sa propre mort. Le fait que Hamlet réussit sa vengeance avant de succomber relève du pur hasard, si bien qu'il rappelle l'intervention arbitraire de l'auteur qui greffe la mort de Cordelia sur l'histoire de King Lear. Décidément, accepter l'invitation à un match amical d'escrime de la part des deux hommes qui ont intérêt à l'éliminer n'a rien de raisonnable.

Quelle idée en fin de compte de terminer une histoire de vengeance par un match d'escrime dont le vengeur devait être la seule victime ! Au juste, c'était l'idée de qui ? Pas de Saxo Grammaticus, ni de Belleforest: dans leurs versions le héros affronte carrément le roi meurtrier. Peut-être de Kyd, mais dans ce cas quel contraste avec la fin éminemment logique de son autre tragédie de vengeance, pour rien dire du modèle de Sénèque. Il y a un seul élément du récit source qui se trouve remanié dans le match d'escrime et qui semble susceptible de l'avoir inspiré : chez Saxo et Belleforest, le héros trompe le roi en substituant à l'épée du dernier la sienne, laquelle on avait attachée à sa fourrure pour l'empêcher de se faire mal dans sa folie supposée. D'où,

226 Cité par Jenkins, Introduction, p. 83.

227 Comme la plupart des critiques récents, j'accepte l'idée que la pièce allemande, Tragoedia der bestrafte Brudermord, dont l'action correspond en général à celle de Hamlet, dérive du premier in-quarto de la pièce de Shakespeare. Voir notamment Jenkins, Introduction, p. 112-22, et Stanley Wells et Gary Taylor, en collaboration avec John Jowett et William Montgomery, William Shakespeare: A Textual Companion, ré-édition avec corrections, New York, Norton, 1997, p. 398 
peut-être, l'échange d'épées au cours du match qui rend possible la vengeance de Hamlet.

Mais ce fameux échange est problématique en soi, à tel point qu'il a suscité beaucoup de commentaires et diverses traditions de mise en scène. Les didascalies du premier in-folio et du premier in-quarto sont loin d'être précises : «In scuffling they change Rapiers »; «They catch one anothers Rapiers, and both are wounded ». Avec " scuffle", l'exclamation du roi, «Part them ; they are incensed» (v.ii.307), vient appuyer l'idée qu'un contact physique se produit d'une façon ou d'une autre, et il est raisonnable de supposer, comme le font la plupart des commentateurs et metteurs en scène, que l'élément provocateur est la blessure de Hamlet qui révèle que la rapière de son adversaire n'est pas mouchetée 228 .

Mais si, au point de vue de la logique « grammaticale» du récit, la conclusion s'avère être un mystère qui en cache un autre, elle remplit en même temps une logique contraire mise en place par la représentation du personnage shakespearien au cours de la pièce. Un match d'escrime dans lequel Hamlet s'engage à contrecœur, servirait de métaphore par excellence pour ses démarches ambiguës vis-à-vis de la tâche imposée par l'esprit de son père, pour le double jeu avec les autres et avec lui-même par lequel il poursuit sa cause tout en se rapprochant de sa ruine. Le véritable match truqué vient donc incarner son déchirement fondamental entre être et non-être, entre l'homme conçu comme «beauty of the world» pour qui l'action est possible, et comme «quintessence of dust», attiré par le néant. Se faire tuer par l'adversaire qu'il a rendu son semblable - «For by the image of my cause I see / The portraiture of his » (v.ii.77-78) - revient à se suicider - son premier souhait, après tout - mais en maintenant le rôle de vengeur engagé. ${ }^{229}$

228 Sur ce point, voir Jenkins, Longer Notes, v.ii.306 s.d., p. 569-70. La didascalie de la pièce allemande, citée dans l'édition de The Riverside Shakespeare, texte établi par G. Blakemore Evans et J. J. M. Tobin, 2e éd., Boston, Houghton Mifflin, 1997, Textual Notes, v.ii.302 s.d., est particulièrement explicite, comme pour un texte destiné à l'usage théâtral. Par contre, le fait qu'elle prescrive en détail un échange sans «scuffling» apparent pourrait indiquer un écart d'une pratique originale non plus comprise ou peu appréciée.

229 J'ai développé ailleurs différents aspects de l'élément auto-destructeur chez Hamlet : voir «Hamlet and Death: A Recasting of the Play within the Player», Essays in Literature, vol. 13, $\mathrm{n}^{\circ} 2$ 2, 1986, p. 201-18; Shakespearean Subversions. The Trickster and the Play-text, Londres, Routledge, 1992, p. 181-85; Self-Speaking in Medieval and Early Modern English Drama. Subjectivity, Discourse and the Stage, Houndmills, Basingstoke, Hampshire, Macmillan, et New York, St. Martin's, 1997, p. 133-38, 267-79. Il est utile de 
Il convient de constater jusqu'à quel point l'auteur des Essais intervient intertextuellement dans les négociations de Hamlet autour de son identité. L'une des premières «agrammaticalités » associées au rôle de vengeur rappelle une faiblesse spécifiquement montaignienne. Lorsque le revenant du père demande à son fils, "Remember me » (I.v.91), le fils déclare spontanément qu'il ne retiendra rien d'autre que ce «commandment» (102) dans «the table of my memory»(98), mais tout de suite fait un geste bizarre en sortant ses vraies tablettes pour écrire. Si cela suggère déjà une peur non seulement d'oublier, mais de voir subvertir sa volonté, cet enchaînement d'idées est confirmé de façon résonante par les paroles de Montaigne se plaignant de sa mémoire peu fiable :

Je ne sçaurois recevoir une charge sans tablettes. [...] Cecy que je sens en la memoire, je le sens en plusieurs autres parties. Je fuis le commandement, l'obligation et la contrainte. Ce que je fais ayséement et naturellement, si je m'ordonne de le faire par une expresse et prescrite ordonnance, je ne le sçay plus faire.

[I cannot receive a charge, except I have my writing tables about me. [...] And what I feele in my memorie, I feele in many other parts of mine. I eschew commandement, duty, and compulsion. What I doe easily and naturally, if I resolve to doe it by expresse and prescribed appointment, I can then doe it no more.] (II, 17, 649-50a ; trad. Florio, $2: 375-76)$

Voilà une première image montaignienne mise en scène pour compliquer le scén ario typique de la vengeance.

Ce mauvais augure intertextuel se réalise à travers les débats que le héros entame avec lui-même au sujet justement de l'héroïsme, qui lui est loin d'être «aisé » et «naturel». C'est encore par un virage montaignien qu'il se dirige vers les modèles ultimes de la vanité de tout effort héroïque face à la mort. Dans le cimetière il permet à son «imagination»-contrairement à l'avis de Horatio «'Twere to consider too curiously to consider so » (v.i.199) - de suivre le «noble dust», d'abord d'Alexandre, puis de César, jusqu'à de bas usages. L'association de ces deux héros est assez répandue, tout comme

considérer le comportement de Coriolan à la même lumière (voir Shakespearean Subversions, p. 195-99), et les propos de Menenius, bien que parlant de lui-même, s'avèrent d'autant plus révélateurs à cet égard : « He that hath a will to die himself fears it not from another » (Cor., Riverside Shakespeare, v.ii.104-5). 
l'allusion à Alexandre comme memento mori ${ }^{230}$. Mais la comparaison étendue des deux héros que fait Montaigne (II, 34) s'est déjà faite sentir dans les délibérations de Hamlet. «[T]o take arms against a sea of troubles / And by opposing end them » (III.i.59-60) évoque, comme nous informent les éditeurs ${ }^{231}$, la pratique connue de guerriers celtiques désespérés, de sorte que l'idée d'action menant à la mort est déjà présente, imbriquée avec le leitmotiv de l'escrime. Un héros peut donc lui aussi ressembler à un torrent d'eau puissant, jusqu'à l'intervention de la raison, après quoi :

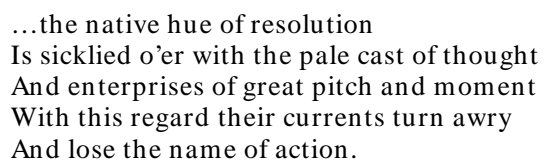

\section{Or, Montaigne estime César :}

un peu plus retenu et consideré en ses entreprinses qu'Alexandre : car cettuy-cy semble rechercher et courir à force les dangers, comme un impetueux torrent qui choque et attaque sans discretion et sans chois tout ce qu'il rencontre.

[somewhat more warie and considerate in his enterprises, than Alexander; for the latter seemeth to seek out, and by maine force to runne into dangers, as an impetuous or raging torrent, which without heede, discretion, or choise, shockes and checkmates what ere it meteeth withall.] (II, 34, 739a ; trad. Florio, 2 : 468)

Même chez César, la «résolution» elle-même peut être ambiguë, cachant comme un désir de «se perdre»: «il me semble lire en plusieurs de ses exploits une certaine résolution de se perdre, pour fuyr la honte d'estre vaincu. [me seemeth I reade in diverse of his exploits, a certain resolution rather to lose himself, then to abide the brunt or shame to be overthrowne]» (II, 34, 740 a ; trad. Florio, $2: 469$ ) - c'està-dire, "to suffer / The slings and arrows of outrageous fortune » (III.i.57-58) ${ }^{232}$. Montaigne continue : «Et quant aux entreprinses qu'il a faites à main armée, il y en a plusieurs qui surpassent en hazard tout discours de raison militaire [And concerning the enterprises he underwent with armed hand, there are divers of them, which in respect

\footnotetext{
230 Voir J enkins, notes à v.i.191, 191-94, 206-9.

${ }^{231}$ Voir Jenkins, p. 491-92.

232 « Abide» rend la traduction ici plus proche du texte shakespearien.
} 
of the hazard, exceed all discourse of military reason] » (ibid.). Outre, encore, le chevauchement du lexique, soulignons la conclusion de Montaigne qu'un tel courage, qui risquerait autrement d'être classé comme la «hardiesse temeraire [rash fond-hardinesse] » (II, 34, 741a ; trad. Florio, $2: 471)$ des jeunes, signale que « [c]es gens là ont eu je ne sçay quelle plus qu'humaine confiance de leur fortune [such men have had a kinde of more than humane confidence of their fortune] » (II, 34, $740 \mathrm{a}$; trad. Florio, 2: 469). Par contre, Hamlet indécis croit que «fortune» lui est « outrageous».

Mais avant qu'il n'accepte l'invitation de Laertes et Claudius, Hamlet ré-imagine sa relation avec la fortune. Il se convainc, comme il l'explique à Horatio, que « rashness» lui a sauvé la vie (v.ii.6-9). Ce changement de perspective est d'autant plus frappant qu'il contraste avec son éloge de Horatio dans le troisième acte. Là il s'agit encore de supporter, de manière stoïque, ce que la fortune peut asséner de mauvais :

thou hast been

As one, in suff'ring all, that suffers nothing,

A man that Fortune's buffets and rewards

Hast ta'en with equal thanks; and blest are those

Whose blood and judgement are so well commeddled

That they are not a pipe for Fortune's finger

To sound what stop she please.

(III.ii.65-71)

Grâce encore à l'intertexte montaignien nous pouvons mesurer plus facilement l'évolution de la pensée du personnage :

Nostre esprit est un util vagabond, dangereux et temeraire; il est malaisé d'y joindre l'ordre et la mesure. [...] Certes il est peu d'ames si reiglées, si fortes et bien nées, à qui on se puisse fier de leur propre conduicte, et qui puissent, avec moderation et sans temerité, voguer en liberté de leurs jugements au delà des opinions commune. [...] C'est un outrageux glaive que l'esprit à son possesseur mesme, pour qui ne sçait s'en armer ordonnément et discrettement.

[Our spirit is a vagabond, a dangerous, and fond-hardy implement; it is very hard to joyne order and measure to it. [...] Verily, there are few soules, so orderly, so constant, and so well borne, as may be trusted with their owne conduct, and may with moderation, and without rashnes, saile in the liberty of their judgments beyond common opinions. [...] The spirit is an outragious glaive, yea even to his owne possessor, except he have the grace, very orderly and discreetly to arme him self therewith.] (II, 12, 559a,b,c ; trad. Florio, 2 : 271-72) 
Hamlet, en décidant que «our indiscretion sometimes serves us well » (v.ii.8), a trouvé un autre modèle, celui d'Alexandre qui « semble rechercher et courir à force les dangers, comme un impetueux torrent qui choque et attaque sans discretion et sans chois tout ce qu'il rencontre [seemeth to seek out, and by maine force to runne into dangers, as an impetuous or raging torrent, which without heede, discretion, or choise, shockes and checkmates what ere it meteeth withall]» - et pourquoi pas, puisque son destin est après tout la poussière ? En adoptant « rash fond-hardinesse» comme son arme, il se révèle armé d'un « esprit» qui est un « outrageux glaive [...] à son possesseur mesme», d'autant plus que sa «folie»a outragé son semblable, non plus Horatio mais maintenant Laertes, jusqu’à mettre en garde la "sagesse » du dernier : "though I am not splenative and rash, / Yet have I in me something dangerous, / Which let thy wiseness fear»(v.i.254-56) ${ }^{233}$. Or, Hamlet a trouvé ce modèle auto-destructeur précisément grâce à un esprit exercé continuellement dans son «discourse of reason», le «continual practice» (v.ii.207) d'escrime intellectuelle qui a remplacé «all custom of exercises » (II.ii.296-97) sous l’influence de sa pensée mélancolique. En quoi il contraste avec Laertes, doué d'un «masterly report / For art and exercise in your defence / And for your rapier most especial» (IV.vii.95-97).

Lorsque Montaigne parle de l'esprit comme un «outrageux glaive », cela fait partie d'un moment exceptionnel à la conclusion de l'« Apologie », un moment de mise en abyme où il se révèle conscient de l'extrémité, même du danger, de donner libre cours à son discours de raison afin de nier la valeur de la raison même. Montaigne s'adresse alors à une femme docte (vraisemblablement Marguerite de Valois ${ }^{234}$ ), en lui conseillant « en vos opinions et en vos discours, autant qu'en vos mœurs et en toute autre chose, la moderation et l'attrempance et la fuite de la nouvelleté et de l'estrangeté. [I persuade you, in your opinions and discourses, as much as in your customes, and in every other thing, to use moderation and temperance, and avoide all newfangled inventions and strangeness.» (II, 12, 558 a ; trad. Florio,

233 Son agressivité n'a donc guère l'effet calmant que peut produire exceptionnellement la «résolution», d'après Montaigne (I, $1,7 \mathrm{a}$; trad. Florio $1: 17$ ) sur ceux qui cherchent la vengeance. À noter aussi qu'il n'assume pas la responsabilité de ses actions, au contraire, lorsqu'il demande pardon à Laertes juste avant le match en blâmant son «madness " (IV.ii.233)

234 Autre candidate : Catherine de Bourbon. Voir Villey, p. 1290, note 35 à la p. 557. 
2 : 271). En poursuivant ainsi la voie moyenne - comme Horatio, sans « consider[ing] too curiously», car Montaigne est d'accord qu'«il ne faict mie bon estre si subtil et si fin [it is not good to be so subtill, and so curious]» (ibid.) - elle pourra «maintenir vostre Sebond par la forme ordinaire d'argumenter dequoy vous estes tous les jours instruite, et exercerez en cela vostre esprit et vostre estude [maintaine your Sebond, with the ordinary form of arguing, whereof your are daily instructed, and will therein exercise both your mind and study] » (II, 12, 557-58a ; trad. Florio $2: 270$ ). Sinon, voici le risque auquel Montaigne s'expose, de même que Hamlet :

car ce dernier tour d'escrime icy ${ }^{235}$, il ne le faut employer que comme un extreme remede. C'est un coup desesperé, auquel il faut abandonner vos armes pour faire perdre à vostre adversaire les siennes, et un tour secret, duquel il se faut servir rarement et reservéement. C'est grand temerité de vous perdre vous mesmes pour perdre un autre.

Il ne faut pas vouloir mourir pour se venger...

[For this last trick of fence, must not be employed but as an extreme remedy. It is a desperate thrust, gainst which you must forsake your weapons, to force your adversary to renounce his, and a secret slight, which must seldome and very sparingly be put in practise. It is a great fond-hardinesse to lose our selfe for the losse of another. A man must not be willing to die to revenge himself...] (II, 12, 558a,b; trad. Florio, $2: 270$ )

Montaigne, connaisseur en escrime, se réfère-t-il à un «tour secret » véritable ? D’une part, les historiens spécialisés dans l'escrime renaissante ne semblent pas connaître une telle manœuvre ${ }^{236}$; d'autre

235 « à savoir de refuser toute valeur à la raison humaine » (Villey, p. 558, note 1).

236 Sur ce point j'ai profité des connaissances approfondies de mon collègue Pascal Brioist. À propos de l'intérêt technique de Montaigne pour l'escrime, voir Pascal Brioist, Hervé Drévillon et Pierre Serna, Croiser le fer. Violence et culture de l'épée dans la France moderne (XVI $-X V I I^{e}$ siècle), coll. "Époques », Seyssel, Champ Vallon, 2002, p. 67-68. Je n'ai trouvé dans la critique montaigniste aucune suggestion qu'il puisse s'agir d'une manœuvre véritable. En tout cas, l'expression reste métaphorique pour E. Limbrick, «"Ce dernier tour d'escrime" ", Cahiers de l'Association Internationale des Études Françaises, 1981 p. 53-64, dont d'ailleurs la conclusion simple que «Montaigne renoncera aux armes de la raison» pour «arriver à la doctrine paulinienne par excellence» (p.64), semble contourner le motif du suicide. Par contre, pour François Rigolot, «D’une théologie "pour les dames" à une apologie "per le donne"?", in Montaigne, "Apologie de Raimond Sebond». De la Theologia à la Théologie, éd. Claude Blum, Études montaignistes, $\mathrm{n}^{\circ} 6$, Paris, H. Champion, 1990, p. 261-90, l'image revêt une signification particulière en tant que stratagème auctorial destiné à exclure le destinataire féminin : «La métaphore militaire, banale en apparence, a donc son importance, et cela d'autant plus que, loin d'être simplement occasionnelle, elle se "file" tout au long de l'adresse à la Princesse» (p. 284). 
part, un vrai «secret» ne se trouverait pas forcément dans les manuels. Il n'en reste pas moins possible que, tout comme la mention des tablettes, cette image servant à concrétiser la pensée de Montaigne ait pris quelque forme concrète sur scène pour représenter un protagoniste qui effectivement veut «mourir pour se venger». Nul doute qu'il s'agit d'un match à rapière et à dague (v.ii.142). Après sa blessure révélatrice, Hamlet pourrait bien se débarrasser d'une de ses armes, ou même des deux, pour arracher l'épée dangereuse des mains de Laertes, qui ramasserait alors celle de Hamlet. Possible aussi que le «coup desesperé» même, le «tour secret», ne soit pas celui de Hamlet. Si Shakespeare suivait la version de Florio, il aurait pu croire, à cause de la douteuse traduction de «auquel» ${ }^{237}$ comme «gainst which », que le « coup desesperé » vient de l'adversaire ; ce serait donc le coup, apparemment soudain et souvent représenté comme tricheur, par lequel Laertes désespéré réussit enfin à blesser Hamlet. Dans ce cas, ce serait par la suite que Hamlet abandonnerait ses armes pour se jeter sur lui. Mais dans tous les cas, la mise en scène d'un Hamlet se désarmant pour se venger, ne serait-elle pas l'étape suivante éminemment logique dans la série de coups avec l'« outrageux glaive à son possesseur mesme» qu'est son esprit? Hamlet résout donc enfin sa «question» un peu comme Alexandre avec le nœud gordien, en mettant à la place d'un choix raisonné une synthèse par laquelle il peut «suffer / The slings and arrows of outrageous fortune» et à la fois «take arms against a sea of troubles / And by opposing end them » (III.i.56-60).

Le deuxième exemple de la méthode que je tiens à illustrer est beaucoup plus simple (comme la plupart des choses en comparaison avec Hamlet), et d'autant plus exemplaire qu'il s'agit d'une pièce ouvertement mélangée, sinon auto-contradictoire, au niveau du genre, la tragi-comédie tardive qu'est The Winter's Tale ${ }^{238}$. De plus, bien qu'il

Toutefois la métaphore est associée dès le début de l'« Apologie » au raisonnement humain en général.

237 C'est-à-dire «par lequel» (Villey, p. 558, note 2).

238 Cette pièce a été rarement rapprochée de Montaigne, et surtout par le biais du débat entre l'Art et la Nature associée à Perdita (Robertson, p. 202; George Coffin Taylor, Shakspere's Debt to Montaigne, Cambridge, MA, Harvard University Press, 1925, p. 55-56). Toutefois, Taylor (p. 16) trouve un parallèle entre la discussion chez Montaigne de la décision de Paulina de rester en vie et le souhait de Hamlet que Horatio n'agisse pas en « antique Roman» (v.ii.346). 
soit encore question des écarts d'une version précédente, dans ce cas-ci cet original existe : la pièce ne cache guère ses racines dans Pandosto (1588), un roman en prose assez connu de Robert Greene. Shakespeare joue même de façon provocatrice avec son public, par exemple en inversant les deux lieux du roman pour situer la Bohême au bord de la mer, en faisant allusion au sous-titre, The Triumph of Time, par le moyen d'un Chœur incarnant le temps, et surtout en acceptant, pour ainsi dire, le défi lancé par Greene à la fin du roman, où ce dernier lie le suicide du protagoniste à une décision auctoriale arbitraire de clore cette «comédie» avec une «tragédie ». La critique justifie sur le plan esthétique les changements les plus significatifs que le dramaturge à effectués, comme par exemple Halett Smith :

The dignified patience of the accused queen, Hermione, makes necessary the presence of some other character to express resistance to Leontes' tyranny, so Shakespeare creates Paulina. [...] In his source, Pandosto, the falsely accused queen does die; but for a happier ending a reunion like that in Shakespeare's earlier Pericles must be brought about $^{239}$.

Une approche intertextuelle, par contre, assume le point du vue des spectateurs, pour qui l'introduction du personnage de Paulina, ainsi que la substitution d'une conclusion «comique» sous forme de l'animation vraisemblable de la supposée statue de la reine Hermione, événement mis en scène par Paulina, auraient relevé de l'« agrammaticalité ».

Or la critique a aussi, bien sûr, fait des liens entre ces éléments et d'autres textes, en l'occurrence des textes fort disparates : d'un côté, une statue qui se vivifie ne peut pas être sans rappeler l'histoire de Pygmalion, notamment chez Ovide; de l'autre, le nom Paulina peut évoquer l'apôtre, donc privilégier le symbolisme chrétien de la «résurrection» de Hermione. Sans écarter de telles associations, il s'agit ici de signaler qu'un lecteur de Montaigne aurait risqué de se trouver interpellé de prime abord dans un dialogue avec les Essais, et d'une façon remettant l'accent sur un thème qui constitue encore un apport shakespearien: l'amour conjugal, les obligations réciproques des époux, et la capacité d'une femme de montrer une fidélité et un courage censés être masculins.

239 Voir son Introduction à l'édition du Riverside Shakespeare, p. 1613. Pour The Winter's Tale, nous citons cette édition. 
La revivification de Hermione après seize ans a beau se charger d'une valeur symbolique quasi-mystique, grâce à un coup de théâtre exceptionnellement puissant, l'héroüne de la pièce n'était jamais morte. Mais il est indispensable à la conversion profonde du mari qui l'avait martyrisée, fou de jalousie déplacée, que son repentir instantané, ainsi que sa confiance qu'elle peut être soignée («Her heart is but o'ercharged, she will recover»[III.ii.150]) paraissent inutiles. Donc Paulina annonce sa mort, tout en invitant le «tyrant» (III.ii.207), avec amertume et sarcasme, à essayer de la faire revivre. En effet, «tyran » est le mot clé, répété avec insistance. Il faudra à Leontes une longue « rééducation », à travers sa souffrance et sous la tutelle de Paulina, qui prend la place de la femme injuriée dans la mesure où elle la garde symboliquement présente. Comme preuve qu'il est digne de voir sa femme décider de lui revenir, Leontes se montre reconnaissant envers celle qui l'a virtuellement transformé de tyran en exemple à la fois de la vertu stoïcienne et de vrai amour. Il annonce le ton de la scène finale et émouvante en s'exclamant « $\mathrm{O}$ grave and good Paulina, the great comfort / That I have had of thee!» (v.iii.1-2), paroles qui résonnent jusqu'à la fin : «O Paulina » (8) ; «O sweet Paulina » (70) ; «O peace, Paulina » (135) ; «Good Paulina, / Lead us from hence» (151-52).

C'est un moment qui aurait pu évoquer une situation aussi chargée d'émotion que Montaigne raconte dans son essai «De Trois bonnes femmes» (II, 34, le chapitre qui suit celui sur César et Alexandre). Là, Sénèque, condamné au suicide par Néron, le tyran par excellence, découvre que sa femme est suffisamment imbue à la fois d'amour et de philosophie pour vouloir mourir avec lui, et Florio embellit les propos du stoïcien en le faisant s'exclamer «O my deare Paulina» $(2: 479)$ - c'est-à-dire Pompeia Paulina. Le but déclaré de cet essai est de fournir des exemples exceptionnels de dévotion conjugale, car «La touche d'un bon mariage, et sa vraye preuve, regarde le temps que la societé dure : si elle a esté constamment douce, loyalle et commode. [The touchstone and perfect triall of a good mariage, respects the time that the societie continueth; whether it have constantly been milde, loyall and commodious] » (II, 34, 744b ; trad. Florio, 2 : 474). Ces exemples contrastent, d'après Montaigne, avec le deuil ostentatoire de la plupart des veuves, dont l'hypocrisie est révélée par leur bonne santé malgré « ces yeux moites [those blubbred eyes] » et «cette piteuse voix [pitty moving voice]» (ibid.). Voilà également 
l'opinion de Hamlet, qui fait référence à l'hypocrisie de sa mère en méprisant «actions that a man might play» tels que «windy suspiration of forced breath » ou « the fruitful river in the eye » (I.ii.7984).

Aussi remarquable dans l'histoire de cette Paulina est le fait que malgré une tentative de suicide elle ne meurt pas. Car le tyran romain, ayant peur des reproches qu'il pourrait s'attirer par la mort d'une si noble femme, la fait soigner, déjà inconsciente, si bien qu'elle revient à la vie ; ces mesures donc, à la différence apparente des « remedies for life » (III.ii.153) commandés par Leontes, s'avèrent efficaces, mais Montaigne nous informe que Paulina est devenue par la suite, dans une conclusion qu'on pourrait qualifier de tragi-comique, un symbole vivant de la vertu et de la souffrance :

Et ce que, contre son dessein, elle vesquit dépuis, ce fut treshonorablement et comme il appartenoit à sa vertu, montrant par la couleur blesme de son visage combien elle avoit escoulé de vie par ses blessures.

[And that afterward, contrary to her intent, she lived, it was very honourable, and as befitted her vertue, shoewing by the pale hew and wanne colour of her face, how much of her life she had wasted by her incisions.] (II, 35, 749a ; trad. Florio, 2 : 480-81)

C'est donc Hermione qui prend, chez Shakespeare, la place de cette Paulina, Hermione qui préférait elle aussi trouver la mort plutôt que de perdre son honneur : " no life / (I prize it not a straw), but for mine honour, / Which I would free...» (III.ii.109-11). Sur le visage de sa «statue » est marquée, en termes du temps perdu, «combien elle avoit escoulé de vie [how much of her life she had wasted]» - l'ineffaçable trace de la tragédie au moment du tournant comique :

Leontes. But yet, Paulina,

Hermione was not so much wrinkled, nothing So aged as this seems. [...]

Paulina. So much the more our carver's excellence,

Which lets go by some sixteen years, and makes her As she liv'd now.

Leontes. As now she might have done,

So much to my good comfort as it is

Now piercing to my soul.

Même en survivant «to [Leontes'] good comfort», et au réconfort de sa fille - Perdita retrouvée - pour qui, dit-elle, « I [...] have preserv'd 
myself»[v.ii.125-28]), Hermione incarne le principe exprimé par Sénèque dans la lettre à Lucilius avec laquelle Montaigne clôt ce chapitre. Ayant expliqué que sa considération pour sa femme l'a déjà motivé de s'abstenir du suicide, le philosophe ajoute :

il faut prester quelque chose aux honnestes affections; et par fois, encore que les occasions nous pressent au contraire, il faut r'appeller la vie, voire avecque tourment. [...] Celuy qui n'estime pas tant sa femme ou un sien amy que d'en allonger sa vie, et qui s'opiniastre à mourir, il est trop delicat et trop mol. [...] il faut par fois nous prester à nos amis, et, quand nous voudrions mourir pour nous, interrompre notre dessein pour eux. C'est tesmoignage de grandeur de courage, de retourner en la vie pour la consideration d'autruy, comme plusieurs excellens personnages ont faicts. [...] Je me suis contrainct à vivre, et c'est quelquefois magnanimité que vivre.

[something must be lent to honest affections, and sometimes, although occasions urge us to the contrary, life must be revoked againe, yea with torment. [...] He who esteemeth not his wife or a friend so much, as that he will not lengthen his life for them, and wil obstinately die, that man is over-nice, and too effeminate. [...] we must sometimes lend our selves unto our friends, and when we would die for us, we ought for their sakes to interrupt our deseigne. It is a testimony of high courage to returne to life for the respect of others as diverse notable men have done. [...] I have enforced my selfe to life: And to live is sometime magnanimitie.] (II, 35, 750a ; trad. Florio, 2 : 481-82)

Si ces paroles auraient bien pu être prononcées par l'héroïne shakespearienne, cela nous rappelle également que Shakespeare, lui, mène jusqu'au bout, à l'aide de Hermione, Paulina et Perdita, le thème annoncé mais finalement détourné par Montaigne : «De Trois bonnes femmes » (c'est moi qui souligne).

Mais tout comme le «tour d'escrime» de l'« Apologie» s'insinue dans Hamlet de manière à transcender la thématique et témoigner d'un «match» au niveau même de la composition, le «doublage» intertextuel de Paulina devient aussi, pour ainsi dire, une affaire entre professionnels. Car Montaigne recommande ses histoires, non seulement aux lecteurs comme matière morale, mais aussi aux écrivains comme dignes d'être incorporées dans leurs ouvrages. Ses propos revêtent une ironie singulière face à un dramaturge qui joue, dès le titre et jusqu'à la conclusion de sa pièce, sur l'improbabilité de l'action (« That she is living, / Were it but told you, should be hooted at 
/ Like an old tale »[v.iii.115-17] ${ }^{240}$ ), et qui s'affaire pour renverser la conclusion tragique du conte vieilli de Greene. Voici Montaigne :

Voylà mes trois contes tres-veritables, que je trouve aussi plaisans et tragiques que ceux que nous forgeons à notre poste pour donner plaisir au commun; et m'estonne que ceux qui s'adonnent à cela, ne s'avisent de choisir plutost dix mille tres-belles histoires qui se rencontrent dans les livres, où ils auroient moins de peine et apporteroient plus de plaisir et profit.

[Loe here my three true stories, which in my conceit are as pleasant and as tragicall, as any we devise at our pleasures, to please the vulgar sort withall : and I wonder, that those who invent so many fabulous tales, do not rather make choise of infinite excellent, and quaint stories, that are found in bookes, wherein they should have less trouble to write them, and might doubtlesse proove more pleasing to the hearer, and profitable to the Reader.] (II, 35, 749a ; trad. Florio, $2: 481$ )

À noter qu'en développant l'aspect de conseil destiné aux écrivains, Florio interpole la mention explicite de «those who invent so many fabulous tales ».

On trouve d'ailleurs chez Montaigne une description de la façon de combiner diverses histoires qui s'applique assez précisément au changement à la fois artificiel et naturel qu'apporte Shakespeare en greffant sur Pandosto la survivance de l'héroïne :

Et qui en voudroit bastir un corps entier et s'entretenant, il ne faudroit qu'il fournit du sien que la liaison, comme la soudure d'un autre metal ; et pourroit entasser par ce moyen force veritable evenemens de toutes sortes, les disposant et diversifiant, selon que la beauté de l'ouvrage le requerroit.

[And whosoever would undertake to frame a compleate and well joynted bodie of them, neede neither employe nor add anything of his owne unto it except the ligaments, as the soldring of another mettall, and by this meanes might compact sundry events of all kindes, disposing and diversifying them, according as the beauty and lustre of the worke should require.] (ibid.)

Si Montaigne entend ainsi, selon sa préférence connue, la valeur supérieure de ce qui est «véritable », à la différence des produits de l'esprit vagabond, il risque sérieusement de miner sa leçon par sa conclusion: «à peu pres comme Ovide a cousu et r'apiecé sa

${ }^{240}$ Cf. les paroles de Mamillius : «A sad tale's best for winter. I have one / Of sprites and goblins » (II.i.24-25) 
Metamorphose, de ce grand nombre de fables diverses [And very neere, as Ovid hath sowen and contrived his Metamorphosis, with that strange number of diverse fables]» (ibid.). Or c'est bien sûr aux Métamorphoses que Shakespeare a emprunté la fable de la statue animée par la force d'amour, pour en faire l'occasion, non seulement de mitiger la tragédie par la comédie mais de «bastir un corps entier» dont la «beauté » est plus «véritable» que la vérité même. Dans la rencontre intertextuelle entre le poète et l'essayiste, n'est-ce pas une façon, sinon d'imposer le dernier mot, au moins de s'approprier avec « grand temerité», désarmé et désarmant, le «dernier tour d'escrime »?

Richard HILLMAN

Université François Rabelais, Tours 